\title{
ANÁLISIS DE LA INCLUSIÓN LABORAL DE LA POBLACIÓN SORDA EN SANTA MARTA
}

\author{
Eder Iván Mendivil-Barceló, ${ }^{1}$ Gustavo Alberto Chinchilla-Rincón, ${ }^{2}$ Náyades Portillo-Mendinueta ${ }^{3}$
}

\author{
${ }^{1}$ Especialista (c), Gerencia de la Calidad y Auditoría en Salud \\ 2 Ingeniero de Sistemas \\ ${ }^{3}$ Magíster (c), Dirección de las Organizaciones del Conocimiento, Universidad Oberta de Cataluña \\ Correo electrónico: nayades.portillo@ucc.edu.co \\ Universidad Cooperativa de Colombia, sede Santa Marta, Colombia
}

Recibido: 30 de enero del 2013. Aprobado: 20 de marzo del 2013.

Cómo citar este artículo: E. I. Mendivil-Barceló, G. A. Chinchilla-Rincón y N. Portillo-Mendinueta, "Análisis de la inclusión laboral de la población sorda en Santa

Marta". Ingeniería Solidaria, Vol. 9, No. 16, pp. 39-48, Dic., 2013.

Resumen. El artículo presentado es producto de la investigación titulada "Herramientas que aporta la Ingeniería de Sistemas para la vinculación laboral de sordos-mudos en la zona urbana del municipio de Santa Marta”. Esta investigación tuvo por objetivo general buscar y analizar herramientas de tipo tecnológico que puedan ayudar a mejorar el desempeño de la población antes mencionada con el fin de proporcionar mayores oportunidades de vinculación laboral. El estudio se llevó a cabo en el periodo de mayo a octubre del 2011, utilizando un diseño descriptivo cuantitativo de corte transversal. El artículo expone resultados acerca del análisis de la ubicación laboral de los discapacitados auditivos en Santa Marta, análisis de nivel aptitudinal de la población estudiada y algunas recomendaciones para mejorar su vinculación laboral. También presenta algunos software disponibles para ayudar en el desempeño laboral de estas personas.

Palabras clave: audición, cofosis, discapacidad, tecnología.

\section{ANALYZING WORKFORCE INCLUSION OF THE Deaf Population in Santa Marta}

Abstract. This article is the product of a research study titled "Tools provided by systems engineering for workforce participation of deaf and/ or mute people in the urban zone of the Santa Marta municipality”. This research looked for and analyzed technological tools that could help improve deaf and/or mute people's performance to give them greater opportunities of finding employment. The study was conducted from May to October in 2011, using a quantitative descriptive cross-sectional design. This article presents the results of the analysis related to the workplaces of hearing-impaired people in Santa Marta, an analysis of the aptitude levels of the population studied, and some recommendations for improving their workforce participation. The article also presents software programs which can help them perform their jobs.

Keywords: hearing, cofosis, disability, technology.

\section{ANÁlise da INCLUSÃo laboral dA POPUlaÇÃo SURda EM Santa Marta}

Resumo. O artigo apresentado é produto da pesquisa intitulada "Ferramentas que a Engenharia de Sistemas contribui para a vinculação laboral de surdos e mudos na zona urbana do município de Santa Marta" (Colômbia). Esta pesquisa teve por objetivo geral procurar e analisar ferramentas de tipo tecnológico que pudessem ajudar a melhorar o desempenho da população antes mencionada a fim de proporcionar maiores oportunidades de vinculação laboral. O estudo se realizou no período de maio a outubro de 2011 e utilizou-se um desenho descritivo quantitativo de corte transversal. Este artigo expõe os resultados sobre a análise da localização de pessoas com necessidades especiais auditivas em Santa Marta, análise de nível atitudinal da população estudada e algumas recomendações para melhorar sua vinculação laboral. Também apresenta alguns softwares disponíveis para ajudar no desempenho laboral dessas pessoas.

Palavras-chave: audição, cofose, pessoas com necessidades especiais, tecnologia. 


\section{Introducción}

La inclusión laboral es posible con herramientas que ayuden y puedan manejar las personas con discapacidad. Donde nuestro recurso, nuestro ser, nuestros valores hacen que esas personas también formen parte de nuestras vidas. El objetivo desde el punto de vista de nuestra carrera de Ingeniería de Sistemas son todas esas herramientas, marcos de trabajo, técnicas y tecnología que ayudarían a apalancar y apoyar a estas personas, para que puedan desarrollar actividades laborales acordes a sus capacidades, tomando como base una formación académica de cualquier orden.

Durante muchos años se ha contemplado a la persona con discapacidad auditiva desde diferentes puntos de vista, influenciados por el acontecer histórico; de tal modo, que en esta última década, entre otras, se reconoce a la persona con esta discapacidad como un sujeto integrante de una comunidad lingüística minoritaria, y desde ello se inserta dentro del planteamiento de la corriente bilingüe (lengua de señas colombiana-española lecto-escrito), la cual permite el desarrollo de su identidad lingüística, cultural, personal y social [1].

Si se considera que tanto la Constitución Política colombiana, como los propósitos del Gobierno, se concentran en buscar la equidad e igualdad de los derechos para tener condiciones que permitan el libre desarrollo de la persona con accesibilidad a los diferentes medios y posibilidades, es importante considerar el reconocimiento de la igualdad como elemento esencial y del respeto a la diferencia [1].

Las personas con audición poseen el grado suficiente para comprender el habla, puesto que sus condiciones auditivas son adecuadas; pueden interpretar el habla sin ayuda de aparatos o técnicas especiales. Las personas que poseen discapacidad auditiva no son capaces de entender el habla. Aunque pueden percibir algunos sonidos, la limitación puede ser tan grave que el individuo no puede distinguir sonido alguno, aun si utiliza aparatos. Estas personas sufren un trastorno de audición y para comunicarse dependen de la vista, sus manos y, en casos en los que físicamente sean necesarios, sistemas de amplificación que les ayudan a interrelacionarse con su entorno.

Entre los distintos tipos de discapacidades auditivas podemos citar: la hipoacusia y la sordera [2]. Según el análisis realizado, se puede decir que las oportunidades de trabajo y el estilo de vida ofrecido a personas discapacitadas de tipo auditivo no es el más óptimo; se ha observado como problemática fundamental o central una discriminación hacia estas personas en el ámbito laboral, lo cual afecta su bienestar como seres humanos dentro de una sociedad y cultura. Partiendo de este paradigma central se originan unas causas y efectos que entran en juego y ayudan a desarrollar esta problemática de tipo social. Dentro de las causas podemos encontrar que actualmente no hay el apoyo suficiente por parte del sector privado o público en la inversión de recursos tecnológicos que les faciliten el desempeño laboral de forma normal dentro de una organización empresarial -se está viendo que las organizaciones o empresas no cuentan con personal capacitado como interpretes para el trato de personas discapacitadas de tipo auditivo; esta problemática no sólo se presenta en organizaciones laborales, sino de entidades gubernamentales que no tienen en cuenta a las personas de este tipo de discapacidad.

En Santa Marta actualmente el único ente educativo de educación superior que está brindando apoyo masivo a la población con discapacidad auditiva es el SENA, que brinda el apoyo a este tipo de personas, ofreciéndoles una formación titulada en mantenimiento de computadores; brindándoles la posibilidad de tener un mejor futuro y estilo de vida. En la parte de educación básica se encuentra CELCA, para la formación primaria, secundaria y media vocacional de jóvenes discapacitados. Si a la población discapacitada no se le brinda oportunidades de desarrollo se le estará violando sus derechos de ser tratados como personas que puedan aportar nuevas ideas a esta sociedad.

\section{Materiales y método}

El estudio se llevó a cabo en el periodo de mayo a octubre del 2011. Se utilizó un diseño descriptivo cuantitativo de corte transversal, que abarca una problemática inmersa en nuestro diario devenir como lo es la falta de herramientas tecnológicas que ayuden a evitar discriminación de las personas con discapacidad, en este caso del tipo auditivo, en el ambiente laboral.

En esta investigación se realizó un tipo de muestreo no probabilístico intencional de las empresas donde tienen vinculadas personas con discapacidad auditiva, las personas que frecuentemente se interrelacionan con este tipo de población y el grupo de adultos discapacitados auditivos que en la actualidad adelanta la titulación en mantenimiento de computadores en el Centro Ecoturístico y Logístico del SENA, regional Magdalena. 


\subsection{Instrumentos}

Para la recolección de los datos se establecieron tres frentes de trabajo. En primer lugar, una muestra intencional con cinco empresas en las cuales hay vinculados discapacitados auditivos, con el fin de establecer las condiciones de trabajo de estas personas en cada una de ellas, los ambientes, herramientas tecnológicas, los roles y la interrelación con los demás trabajadores. Un segundo frente fue el grupo de 18 estudiantes con discapacidad auditiva de la titulación de mantenimiento de computadores del SENA, regional Magdalena, para establecer el nivel aptitudinal de esta muestra frente a la formación que se encuentran recibiendo. Un último frente fue el grupo de personas de CELCA, que continuamente trabajan entre otras poblaciones con los discapacitados auditivos que realizan sus estudios de básica primaria, secundaria y media vocacional en esa institución de educación inclusiva en el municipio de Santa Marta.

Las encuestas utilizadas fueron estructuradas con respuestas de selección múltiple, las cuales, tras ser aplicadas al grupo de estudiantes de la titulación de mantenimiento de computadores, se verán en el capítulo de anexos. Se contó con la ayuda de una intérprete de lenguaje de señas debido al desconocimiento de los investigadores de este tipo de lenguaje. También se realizaron entrevistas a diferentes personas que han estudiado esta problemática en Santa Marta.

Las demás encuestas aplicadas se realizaron para ser resueltas de forma individual por los administradores o gerentes de las empresas visitadas y por el personal docente y de apoyo de CELCA.

\section{Estado del arte}

Esta investigación fue iniciada con sentido de pertenecía y de preocupación de la situación actual de las personas discapacitadas. Se observa que el estilo de vida y las oportunidades de tipo laboral son muy pocas dentro de esta sociedad; se está dejando de lado estas personas, discriminando sus capacidades aptitudinales, los cual afecta su desarrollo personal.

Discapacidad es un término general que abarca las deficiencias, las limitaciones de la actividad y las restricciones de la participación. Las deficiencias son problemas que afectan a una estructura o función corporal; las limitaciones de la actividad son dificultades para ejecutar acciones o tareas, y las restricciones de la participación son problemas para participar en situaciones vitales. Por consiguiente, la discapacidad es un fenómeno complejo que refleja una interacción entre las características del organismo humano y las características de la sociedad en la que vive [3].

La discapacidad es uno de los factores limitantes del estilo de vida de muchas personas, no se está pensando como una sociedad integral en la que se incluyan todo tipo de individuos, sólo se tiene en cuenta aquellas personas que no poseen limitantes, provocando un desequilibrio en las oportunidades brindadas para un desarrollo aptitudinal.

El punto de referencia para tratar es la discapacidad auditiva. Se puede decir que una persona discapacitada de este tipo es aquella que posee limitantes en el sentido de la audición, el cual no puede percibir ninguna señal acústica o sonora. En la actualidad se le ha venido dando una mala terminología a esta situación.

La sociedad ha definido como sordomudas a las personas sordas. Responde a la idea de una supuesta incapacidad de las personas sordas para comunicarse por medio de una lengua. Mediante una educación adecuada puede acceder a la lengua oral en su forma escrita $y$, en función de las circunstancias individuales, hablada. Por tanto, la expresión "mudo" es incorrecta al igual que el término sordo [4].

Se han realizado varias investigaciones que apoyan la temática que se va a tratar sobre la discapacidad y nos brindan apoyo para el desarrollo de este proyecto de investigación. En la Universidad Pedagógica Nacional se elaboró una investigación sobre comunicación aumentativa y alternativa mediante tecnologías de apoyo para personas discapacitadas, que tiene como objeto de estudio la comunicación de estas personas con deficiencias. Se pretende alcanzar una mejor solución a estos problemas comunicativos mediante procesos de investigación y de proyección social, para generar una propuesta pedagógica apoyada en las tecnologías de la información [5].

Dentro del margen se ha podido observar que en otras ciudades se ve la preocupación por el vivir de estas personas discapacitadas, donde se realizan investigaciones y proyectos para mejorar su estilo de vida. En el Distrito no se ha visto gran interés de preocupacion por este grupo de personas. En Bogota, la Universidad del Rosario hace el aporte con el proyecto Tecnologia y discapacidad, con el cual se busca la implementación de la Política Sectorial en Salud para la Prevención y el Manejo de la Discapacidad en Bogotá. El objetivo es la formulación de los lineamientos para el otorgamiento de ayudas 
técnicas por los bancos de ayudas técnicas (BAT) de Bogotá, de manera que se unifiquen los mecanismos y procedimientos para el otorgamiento de estas ayudas, para que tanto profesionales como personas con discapacidad cuenten con herramientas que aseguren un otorgamiento de las ayudas desde el ejercicio de los derechos ciudadanos [6].

Existe un gran número de tecnologías diferentes dentro del conjunto de tecnologías de ayuda. Así, por ejemplo, según las áreas de ejecución, Cook y Hussey (o.c.) describen por lo menos las siguientes:

- Sistemas aumentativos y alternativos de comunicación.

- Tecnologías para la movilidad personal.

- Tecnologías para la manipulación y el control del entorno.

- Ayudas sensoriales para personas con discapacidad visual, auditiva o táctil [7].

Dentro de los anteriores descritos, este proyecto suma una ayuda técnica más: Tecnologías de acceso al ordenador (Adaptative Technology), que sería todos los sistemas (hardware y software) que permiten a personas con discapacidad física o sensorial utilizar los sistemas informáticos convencionales [7].

\subsection{Tecnología de apoyo para las personas en situación de discapacidad}

La norma UNE EN Iso 9999 del 2007, que sustituye el término "Ayudas Técnicas" por "Productos de Apoyo", la define como "Cualquier producto (incluyendo dispositivos, equipos, instrumentos, tecnologías y software) fabricado especialmente o disponible en el mercado, para prevenir, compensar, controlar, mitigar o neutralizar deficiencias, limitaciones en la actividad y restricciones en la participación" [8].

Es aquí donde entramos el campo de acción como ingenieros, buscando soluciones óptimas para el mejoramiento del vivir de las personas y de la sociedad en que vivimos, buscando un bien equitativo. Por medio de tecnología de la información (TI) como apoyo fundamental a este tipo de personas discapacitadas, se les brindarán herramientas tecnológicas como facilitadoras de procesos laborales.

El fin de estas herramientas de tipo tecnológico es ayudar a surgir a este tipo de personas discapacitadas convirtiéndose en una gran ayuda y apoyo para el desarrollo y la formación. Estas utilidades les permitirán realizar cargos en diversos roles, no sólo como lo que se encuentra ocurriendo con este tipo de personas que sólo se desarrollan como trabajadores de oficios varios, negándoles la oportunidad de ejercer algún puesto primordial dentro de una organización. La tecnología en general ofrece múltiples instrumentos y funciones cuyo objetivo final ha sido facilitar la vida de las personas e incrementar la eficacia de sus actividades.

Las nuevas herramientas tecnológicas pueden hacer posible que muchos trabajos tradicionales puedan realizarse de una forma más sencilla por las personas con discapacidad, con lo que abre el acceso al empleo existente. Por otro lado, porque el desarrollo de la sociedad de la tecnología de la información está haciendo nueva formas de actividad económica, y con ello nuevas formas de trabajo [9].

Las TIC han logrado un auge en protagonizar el desarrollo de muchas actividades y como apoyo de muchos procesos. Dentro de estos encontramos ayudas a las personas discapacitadas, en las que se pueden ver en diversos proyectos de investigación tanto a nivel nacional como a nivel mundial. En la parte mundial, se ha visto un gran apoyo a este tipo de personas. Unos ejemplos que cabe resaltar podrían ser el uso de una aplicación de IPhone que permite trabajar a una persona discapacitada de tipo auditiva como cartero del grupo Gureak.

Gureak explica que el usuario coloca el altavoz del iPhone en el timbre, la aplicación detecta que se ha producido un sonido y muestra en la pantalla la gráfica con la onda detectada. También indica a través de un botón verde y con un sonido de voz que se ha producido una respuesta. En ese momento, el usuario puede pulsar el botón 'reproducir' para poner en marcha un mensaje pregrabado que solicita la apertura de la puerta.

La aplicación funciona de manera autónoma y no es necesario estar conectado a la red para que se ejecute, por lo que el dispositivo que se use no es necesario que disponga ni de conexión a la red de datos, ni tarjeta sim con tarifa de voz. Desde el pasado mes de mayo ${ }^{1}$, Itok se está utilizando diariamente en EgunOn con resultados muy satisfactorios [10].

Otro ejemplo se basa en un proyecto de origen japonés.

La empresa japonesa NTT DOcomo, subsidiaria de NTT Communications, está trabajando en el desarrollo del primer teléfono que lee los labios, según informó la re-

${ }^{1}$ Según el artículo citado, se refiere a mayo del 2011 
vista New Scientist. "Aunque todavía falta mucho desarrollo, este teléfono podría terminar con los gritos de los usuarios por sus aparatos, hasta en lugares ruidosos. Todo lo que tienen que hacer es modular sus palabras en silencio, y el teléfono las convierte en palabras o texto", señaló la publicación. Los ingenieros de NTT DoCoMo han realizado un prototipo del aparato, y esperan poder comercializarlo en el plazo de cinco años. Sensores de contacto ubicados cerca del lugar por donde se habla en el teléfono detectan señales eléctricas enviadas por los músculos alrededor de la boca. Por otro lado, un sintetizador de habla convierte las señales en palabras o texto para un mensaje o correo electrónico [11].

Uno de los proyectos más innovadores actualmente es Maya es la primera traductora simultánea en lenguaje de signos desarrollada en 3D capaz de reproducir 1.600 signos. Desarrollado por la empresa cordobesa $\mathrm{Xu}$ ha sido ideada para su integración en páginas web, con el fin de facilitar la accesibilidad a un millón de personas sordas en España. El sistema también puede integrarse en teléfonos móviles. Recientemente, Maya ha sido exportada al móvil, convirtiéndose así también en el primer diccionario de lenguaje de signos del mundo con avatar en tres dimensiones [12].

También se encontró en la web un texto elaborado por el Ministerio de Comunicaciones de Colombia sobre el tema de la discapacidad. En este se resalta la importancia de que las instituciones educativas construyan entornos educativos amables para los estudiantes con limitaciones visuales, auditivas o físicas.

La importancia de sistemas de información para la inclusión laboral de las personas discapacitadas es de gran importancia, ya que con la selección de tecnologías, se busca dar respuesta a la demanda de productos, instrumentos, equipamientos o sistemas técnicos para su uso, el de sus familiares, o de los profesionales que los atienden, generada por la población con discapacidad de nuestro país, para evitar compensar, mitigar o neutralizar la discapacidad y mejorar su autonomía personal facilitando su comunicación y el acceso a la información. Así mismo, se busca una mayor integración social y participación activa en la vida económica y laboral, encontrando un sistema de adquisición e implementación de tecnologías, que permita a la población con discapacidad de bajos recursos acceder a ellas. Se recomienda una especial atención en los productos, instrumentos, equipamientos o sistemas técnicos de novedad nacional y de óptima calidad, que cumplan las condiciones de idoneidad, adaptabilidad y funcionalidad que suplan las necesidades creadas por los usuarios, sus familiares y los profesionales en el área de la discapacidad [13].

\section{Resultados}

\subsection{Análisis de datos}

El marco de análisis de esta investigación se determinó según el logro de los objetivos del estudio, teniendo en cuenta el tipo de diseño y las características de los instrumentos que permitieron hacer la descripción de lo que se midió para dar respuesta a la pregunta de investigación. De tal manera que se presentan los resultados, primero sobre la ubicación laboral de los discapacitados auditivos en Santa Marta, segundo, el nivel aptitudinal de la población y, por último, la disposición de esta población frente a la problemática laboral. Las respectivas tabulaciones y análisis de los datos se desarrollaron por Microsoft Excel, ya que nos brinda diversas herramientas de contenido estadístico que nos apalancan en procesos de ordenamiento y sistematización de la información recolectada por medio de los instrumentos antes expuestos.

\subsection{Principales hallazgos}

La situación laboral de este tipo de población discapacitada en el municipio es muy limitada, su mayor vinculación se da en la empresa privada sin que tengan mayor relevancia en la empresa pública. De acuerdo con lo indagado, la vinculación laboral de los discapacitados auditivos en el municipio no alcanza el 10\% de las empresas y dentro de estas su porcentaje de participación es casi nulo.

Esta problemática se puede reflejar en la muestra seleccionada. Sólo un 17\% labora de manera informal y un $83 \%$ no tiene un desarrollo laboral como lo puede mostrar la figura 1. Los factores que inciden en estos resultados son productos de una serie de circunstancias que van desde el ámbito familiar hasta las situaciones sociopolíticas de nuestro entorno.

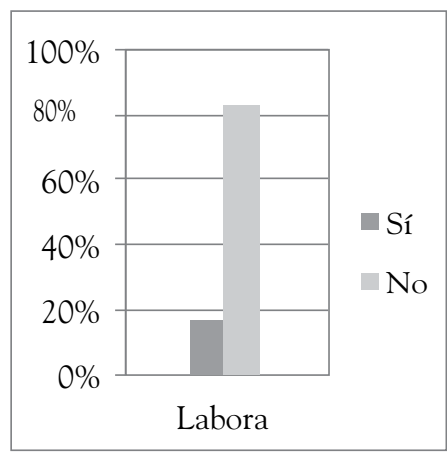

Figura 1. Labora actualmente

Fuente: los autores 
El resultado anterior indica una proporción bastante baja en la incorporación de esta población en el ámbito laboral. Los factores que se asocian a este resultado tienen diferentes fuentes, entre las cuales se pueden citar:

- Falta de apoyo gubernamental

- Bajo nivel de capacitación, técnica o profesional

- Limitación por parte de los familiares

- Falta de interés por parte de entidades para contratación de personas de este tipo de discapacidad.

El concepto general con respecto a la labor que realizan en las diferentes empresas converge en un mismo punto: las personas con discapacidad auditiva son calificados como excelentes trabajadores que cumplen a cabalidad sus funciones, de hecho en la empresa Casa-Limpia tienen vinculada desde hace más de cinco años a una persona con este tipo de discapacidad y es considerado por el administrador como su persona de confianza, llegando a ser el encargado cuando el administrador no puede estar presente en la institución.

Este bajo índice corresponde a la falta de confianza que se tiene en las personas con esta discapacidad. La limitación les rotula ante el común de las personas "oyentes" como una población sin posibilidad de realizar labor que implique un alto grado de responsabilidad. Esta subvaloración se encuentra arraigada no sólo en las personas sin discapacidad auditiva, sino en los miembros de la población en mención, los cuales consideran que las funciones que ellos pueden realizar se limitan a oficios varios dentro de un empresa o en labores artesanales cuando se desempeñan como trabajadores independientes, bien sea haciendo parte de empresas cooperativas o familiares; estas últimas son creadas por los propios familiares para ofrecerles un medio de sustento y actividad que les permita involucrarse con el entorno social y puedan salir de ese aislamiento en el cual la misma sociedad los encasilla y ellos como población partícipe lo permiten, como se aprecia en la figura 2. En esta figura se puede observar en la muestra seleccionada que es nulo el valor de una persona discapacitada quiera montar su propia empresa, con un $0 \%$; se muestra que más de la mitad de la muestra seleccionada, con un $67 \%$, preferirían trabajar en empresas de tipo familiar, evitando el rechazo o la discriminación laboral, y parte, con un 33\%, se sienten con confianza de llegar a una empresa y desempeñarse de manera adecuada. Como consecuencia a estos índices, se pueden ver influidos por la falta de preparación de tipo educativa que le proporcione una confianza al personal discapacitado de desempeñarse en cualquier rol, $\mathrm{y}$ falta de apoyo del entorno laboral.

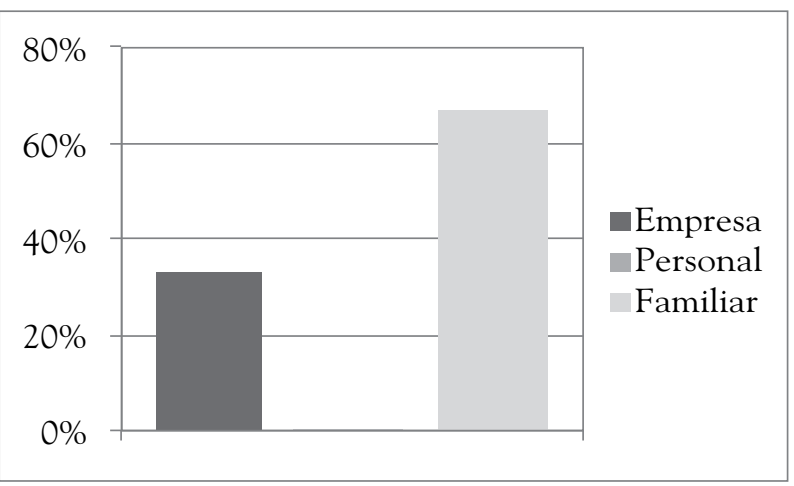

Figura 2. Tipo de empresa a las que les gustaría vincularse Fuente: los autores

La inversión social en esta población se limita al político de turno, a la circunstancia política nacional o internacional vigente; acciones estas que sólo aportan situaciones temporales y condicionadas, que no solucionan la problemática laboral reinante. Todos los factores anotados en el resultado de la figura 2 prevalecen en el resto de resultados obtenidos. En este caso se obtuvo una alta proporción de vinculación a empresas familiares debido a la carencia de incentivos en el mercado laboral, que permitan el acceso a los discapacitados auditivos tener fácil acceso a una empresa bien del sector público o privado.

Actualmente, en estas empresas no se cuenta con intérpretes de lenguaje de señas. Los trabajadores que interactúan con ellos en las empresas se refieren a la forma de comunicarse como "un conjunto de señas que han creado de forma particular para poder relacionarse con ellos", desconociendo el Lsc. Llegan a manejar empíricamente algunas señas que les facilitan situaciones, utilizando la mayoría de las veces tableros mediante los cuales establecen comunicación con estas personas.

Los aportes tecnológicos en las empresas visitadas se circunscriben a la utilización de mensajes de texto soportado con un $17 \%$ de utilización por parte de personal de empresas, mediante teléfonos celulares, como los "recursos o herramientas tecnológicas" para comunicarse con estos empleados (figura 3). A la vez hacen usos de recursos manuales entre los que tenemos tableros, con un 33\%, para la comunicación, una representación nula de profesionales como interpretes, con $0 \%$, y, con un $50 \%$, el uso de señales propias, donde la mitad se basa en recursos propios para lograr comunicación con el personal dis- 
capacitado. Se enfatiza en que el servicio de mensajería es asumido por las personas que los envían y reciben, es decir, procedimientos que no son soportados económicamente por las empresas y por lo tanto no son incluidos como gastos de un presupuesto para facilitar las labores empresariales. No existen en el presente proyectos dentro de estas empresas que faciliten el mejoramiento de las condiciones laborales existentes; en algunos casos sugieren los administradores la creación de herramientas que permitan saber si el empleado entendió la orden, indicarle que lo solicitan, advertirles situaciones; pero estas sugerencias no son plasmadas en "planes de mejoramiento continuo" que permitan no sólo el bienestar laboral, sino también la vinculación de otros discapacitados auditivos, tomando como base la responsabilidad con la cual desarrollan sus labores.

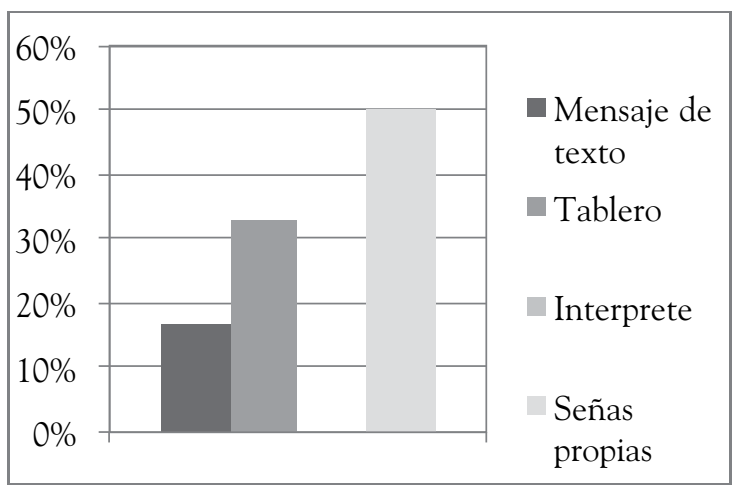

Figura 3. Formas de comunicación utilizadas Fuente: los autores

El segundo objetivo buscó precisar el nivel aptitudinal de las personas discapacitadas en Santa Marta. Al respecto se encontró que la mayoría de la población estudiada no tiene un buen nivel de formación educativa, $\mathrm{y}$ en especial presentan dificultades en las competencias lecto-escrituras. Es preocupante, como lo enseña el gráfico No. 5, que sólo algunos planteles educativos oferten programas educativos dirigidos a esta población, aunque se encuentran experiencias valiosas de apoyo educativo a personas sordas.

En el ámbito educativo se tiene conocimiento de una discapacitada auditiva en la Universidad del Magdalena, que se encuentra cursando segundo semestre en la Licenciatura en Informática, formación a la que accedió luego de un proceso de derecho de igualdad debido a la ausencia de un intérprete en la presentación del examen de admisión. El grupo que está realizando la titulación en el sENA tiene amplias expectativas de vinculación laboral una vez culminen su formación. Este tipo de proyectos permite cambiar la actitud de los integrantes de esa población frente a la sociedad, y les posibilita proyectar en sus familiares otras condiciones, como es el caso del primer egresado discapacitado auditivo del SENA, cuyo hijo ha ocupado cargos públicos destacados.

Este proyecto, que se está realizando a través del SENA, hace parte de la política estatal de ayuda y apoyo a la población discapacitada, en donde no sólo les han brindado oportunidad de formarse como tecnólogos, sino también en el conocimiento de aplicaciones informáticas como: Excel, Access, PowerPoint y Corel Draw, mediante cursos cortos con los cuales esta población se beneficia y aumenta sus posibilidades de acceso al mercado laboral. Para cualquiera de estos proyectos estatales existen limitantes de fondo. Tal es el caso del nivel de comprensión lectora y escritura de esta población, lo cual ralentiza los procesos de enseñanza y aprendizaje. Es frecuente encontrar dificultades cuando se trata de hacerles entender conceptos, que para una persona "oyente" son muy simples y para ellos se tornan difíciles de entender debido a la baja capacidad en lecto-escritura.

La mayoría del personal discapacitado presenta un nivel muy regular del manejo del castellano como se puede ver en la figura 4 , en la cual se nota que más de la mitad de la muestra seleccionada presenta un $67 \%$ de nivel regular para el uso de competencias escritas y lectoras; se presenta un muy bajo índice de que alguien presente un excelente manejo con una representación de un $5 \%$, que es casi insignificante, y con un $28 \%$, personas que se encuentran en un nivel intermedio. Todo esto se debe a la falta de apoyo a este tipo de personas para que alcancen un buen desarrollo, que ayude aumentar sus capacidades, donde no sea sólo un 5\% del personal que maneje buenas prácticas lectoras y escritas, y así alcanzar un mejor desarrollo y tener un mejor nivel aptitudinal para su vinculación laboral.

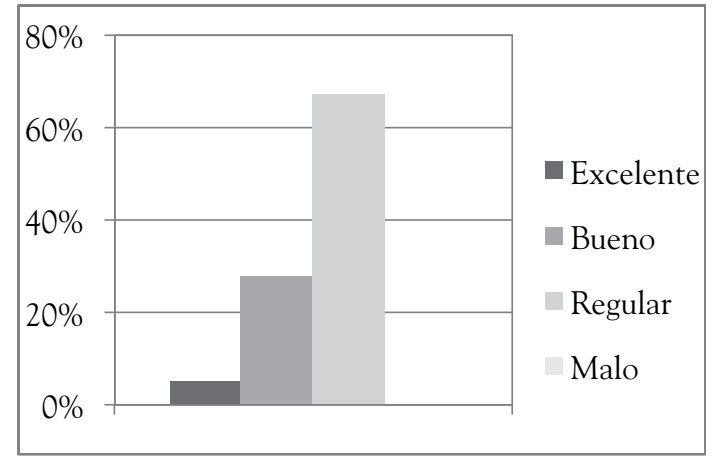

Figura 4. Conocimiento del castellano

Fuente: los autores 
El desconocimiento por parte de los empresarios de los beneficios que se obtendrían con la vinculación de personas con discapacidad es bastante alto. Se precisa que esta vinculación en las empresas se debe a recomendaciones familiares antes que por solicitud directa. Este tipo de vinculación ubica al discapacitado, bien sea auditivo o de otro tipo, en el nivel de servicios generales y no en otras áreas de trabajo dentro de las empresas. Esta condición de discapacidad les asocia una baja autoestima originada por el trato que se les brinda en las empresas. Hasta la presente, en Santa Marta es casi nula la cantidad de personas discapacitadas auditivas con nivel de formación técnica, tecnológica o profesional. Se espera un aumento de las personas discapacitadas auditivas en las diferentes empresas del municipio a partir segundo semestre del 2012, en el área de sistemas, producto del grupo que se encuentra realizando la titulación en mantenimiento de computadores en el SENA.

Que nos muestran sus expectativas de trabajo para el forjamiento de un futuro prometedor, más de la mitad de la muestra con un $64 \%$ los muestra como técnicos en mantenimiento, un $12 \%$ con vocaciones de deportistas, y con un $6 \%$ en varias actividades como cocina, logística, actividades manuales y oficios varios.

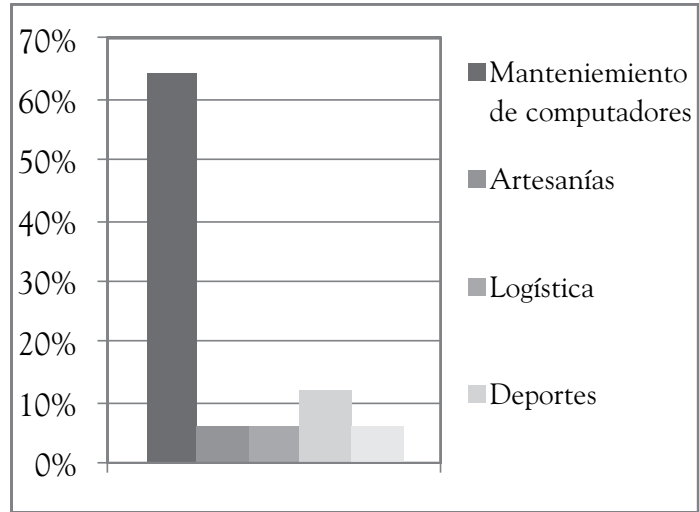

Figura 5. Expectativas de trabajo

Fuente: los autores

El tercer objetivo planteado para la investigación pretendía realizar un análisis de herramientas de software para apoyar el desempeño laboral en Santa Marta. Lastimosamente, después de mucho indagar nos encontramos que en esta ciudad no se utiliza este tipo de software ni empresas que en la actualidad lo utilicen. Un caso ejemplar es España, uno de los países pioneros en desarrollar software e investigación para ayudar a personas con discapacidad auditiva. Detectamos una necesidad y un campo de investigación futura para realizar trabajos de investigación que desde la Ingeniería de Sistemas mejore la calidad de vida de esta población.

\section{Discusión}

Dentro del estudio realizado se pudieron encontrar diversos resultados haciendo cumplimientos a los objetivos anteriormente planteados para el desarrollo de esta investigación. En cuanto al primer objetivo, que es determinar las diversas herramientas que se utilizan en el entorno laboral, se pudo observar que actualmente en el municipio las empresas tanto públicas como de privadas no cuentan con herramientas tecnológicas que permitan la vinculación y el desarrollo laboral y aptitudinal de las personas discapacitadas de tipo auditivo; el apoyo laboral es nulo, lo cual muestra un bajo índice laboral de este tipo de personas.

\section{Conclusiones}

En la utilización de herramientas apoyadas en la ingeniería de sistemas, objeto de este estudio, se logró concluir que estas no se utilizan en ninguna de las empresas encuestadas debido a diversas circunstancias, entre las cuales cabe mencionar:

- Desconocimiento de herramientas o procedimientos que puedan ser aportados a través de la ingeniería de sistemas para el mejoramiento de las labores realizadas por los empleados con discapacidad auditiva.

- Los diversos procedimientos utilizados para la comunicación con los discapacitados auditivos son "suficientes" o acomodados debido a las funciones que realizan.

- Los factores que influyen en la contratación de un discapacitado auditivo se limitan a recomendaciones de tipo familiar; no los contratan por su capacidad laboral o conocimiento particular.

- Consideran estas ayudas tecnológicas como herramientas utópicas y demasiado costosas, que no compensarían ser utilizadas para tratar con una sola persona.

- Desconocimiento de las diferentes normas, leyes y proyectos estatales que les otorgan beneficios tributarios y tecnológicos a las empresas que tengan en su planta de personal empleados con discapacidad auditiva. 
- Desconfianza total en la capacidad laboral de personas con este tipo de discapacidad; se puede tomar esta variable como elemento discriminatorio a nivel personal. Esta discriminación vulnera los derechos básicos de esta población.

Con respecto a la ubicación laboral, las expectativas se fundamentan en el apoyo que las empresas pueden brindar al grupo que realiza la titulación en mantenimiento de computadores en el sENA. Hasta la presente, la mano de obra que esta población aporta a las empresas es utilizada únicamente en labores de servicios generales. Se espera una gran acogida con este primer grupo que egresa a mediados del 2012, como resultado de esta formación. La posibilidad no se limita al trabajo que ellos puedan realizar, sino al cambio de aptitud de otros miembros de la comunidad de discapacitados auditivos frente al estudio y al mejoramiento de la calidad de vida.

La utilización de estas herramientas depende de la divulgación por parte de las entidades gubernamentales, en especial las del sector educativo, para crear en los empresarios una conciencia "inclusiva" que reconozca en la población con este tipo de discapacidad un reglón importante en la fuerza laboral que puede utilizar en las empresas.

\section{Recomendaciones}

\subsection{Recomendaciones a nivel educativo}

- Fortalecer las instituciones "inclusivas", no sólo con su denominación sino con el apoyo de profesionales, intérpretes, modelos lingüísticos, para ampliar la cobertura actual que limita a celca. Aunque existen en el papel más de cien instituciones con el rotulo de "inclusivas", únicamente celca cumple con esta finalidad. Uno de los aspectos que inciden en esta desidia en las demás instituciones por prestar debidamente sus servicios es la deficiente contraprestación económica que les asignan a los profesores o personas calificadas para el manejo y la educación de este tipo de población.

- Crear en el discapacitado auditivo la necesidad de utilizar el LSC como lenguaje propio para que las empresas donde los vinculen tengan la necesidad de utilizar interpretes o aprender mediante capacitaciones en este lenguaje. Esta sensibilización permitirá la vinculación laboral en mayor cantidad de este tipo de discapacitados, y no sólo a discapacitados sino a personal calificado en el manejo de LSC, el cual podría brindar sus servicios a las empresas que cuenten con un número significativo de discapacitados.

- Mejorar las oportunidades educativas en las instituciones de nivel superior, tecnológicas públicas o privadas, para facilitar el acceso a la capacitación de la población discapacitada auditiva, y entregar al mercado laboral una mano de obra calificada tan competente como la de las personas que no sufren de algún tipo de discapacidad.

\subsection{Recomendaciones a nivel tecnológico}

- Implementar en los diferentes ámbitos educativos el desarrollo de proyectos productivos encaminados a facilitar la interrelación con la población discapacitada auditiva, no sólo a nivel laboral.

- Fomentar en los estudiantes de pregrado de las diversas instituciones de nivel superior en el programa de Ingeniería de Sistemas el desarrollo de aplicaciones orientadas a la población con discapacidad auditiva.

- Crear en las instituciones de nivel superior y en instituciones educativas de secundaria semilleros de investigación orientados al desarrollo de proyectos para ayuda a la población discapacitada; establecer los correspondientes nexos con el ente gubernamental y obtener recursos con los cuales se puedan desarrollar o implementar herramientas tecnológicas para mejorar las condiciones de adaptabilidad de la población discapacitada en los diferentes órdenes de vida.

- Tomar ejemplos de mejores prácticas en otras regiones o países e implementarlas en el Distrito, para mejorar en acceso al mercado laboral por parte de los discapacitados auditivos. Estas implementaciones tecnológicas en algunas oportunidades requieren de herramientas o asesoría que no se encuentran fácilmente en cualquier lugar, y son de fácil consecución en las instituciones de educación superior.

\section{Referencias}

[1] P. A. Medina Ríos y M. L. Rincón Bustos, "Plataforma web para aplicaciones tecnológicas que faciliten el aprendizaje escolar y laboral en personas sordas y con deficiencia auditiva. Una construcción interdisciplinar: 
Ingeniería de Software y Fonoaudiología”. Arete, pp. 150-158, 2007. [En línea]. Disponible en: http://www. iberoamericana.edu.co/images/revista_arete_7.pdf

[2] Discapacidad Colombia, Discapacidades, 2009. [En línea]. Disponible en: http://www.discapacidadcolombia.com/modules.php? name $=$ Content $\&$ pa $=$ showpage \&pid=2.

[3] Oranización Mundial de la Salud, Discapacidades, 2011. [En línea]. Disponible en: http://www.who.int/topics/ disabilities/es/.

[4] M. A. Jimenez y G. W. Arregui, Diseño e implementacion de un sistema de generacion de mensajes auditivos para ayudar a personas discapacitadas auditivas y el habla, tesis de grado, Ingeniería Electrónica y Computación. Escuela Superior Técnica de Chimborazo: Riobamba, 2009. [En línea]. Disponible en: http://dspace.espoch. edu.ec/bitstream/123456789/182/1/38T00170.pdf

[5] N. Salazar, Y. Ferrer y I. Toro, Comunicación aumentativa y alternativa mediante tecnologías de apoyo para personas con discapacidad. [En línea]. Disponible en: http://www.pedagogica.edu.co/storage/ted/articulos/ ted13_13arti.pdf.

[6] A. Rios Rincon, R. Laserna Gutierrez, R. Melo Oliveira, M. Vargas y N. Ramirez, Tecnología y discapacidad, Universidad del Rosario-Secretaría Distrital de Salud: Bogota, 2005.

[7] A. M. Ferrer Manchón y F. A. María, "Capítulo 3.2: Ayudas técnicas para estudiantes con discapacidades físicas y sensoriales: las tecnologías de ayuda", Valencia: Universidad de Valencia, 2005. [En línea]. Disponible en: http://ardilladigital.com/DOCUMENTOS/ EDUCACION\%20ESPECIAL/ACCESIBILIDAD\%20
Y\%20AYUDAS\%20TECNICAS/AYUDAS\%20TECNICAS/Ayudas $\% 20$ tecnicas\%20estudiantes\%20discapacidad\%20fisica\%20y\%20sensorial\%20-\%20Ferrer\%20 y\%20Alcantud\%20-\%20art.pdf

[8] Discapacidad Colombia, Tecnología de apoyo para las personas en situación de discapacidad, 2009. [En línea]. Disponible en: http://discapacidadcolombia.com/modules.php?name $=$ Content $\&$ pa $=$ showpage $\&$ pid $=141$

[9] A. J. Lara, "Nuevas teconologias y dispacidad" en Los procesos de inclusion y exclusion de las personas con discapacidad, Madrid: Caritas españolas, 2003, pp. 91-107. [En línea]. Disponible en: http://www.caritas.es/imagesrepository/CapitulosPublicaciones/480/Capitulo\%20 06_08_09_2008_14_11_22.pdf

[10] Vadejuegos.com, Una aplicación para iPhone permite a personas con discapacidad auditiva trabajar como cartero para Grupo Gureak, 2011. [En línea]. Disponible en: http://www.vadejuegos.com/noticias/2011/08/09/ una-aplicacion-para-iphone-permite-a-personas-condiscapacidad-auditiva-trabajar-como-cartero-paragrupo-gureak-101752.html

[11] Y. Soundy, Avances tecnológicos a favor de personas con discapacidad. [En línea]. Disponible en: http://www.sitiodesordos.com.ar/avances\%20tecnol.htm.

[12] Tendencias21, Crean la primera traductora virtual en lenguaje de signos, 2012. [En línea]. Disponible en: http://www.tendencias21.net/Crean-la-primera-traductora-virtual-en-lenguaje-de-signos_a10765.html.

[13] Eduteka, Las tic y el acceso a la información para personas con discapacidad, Jun., 2003. [En línea]. Disponible en: http://www.eduteka.org/Discapacidad1.php. 